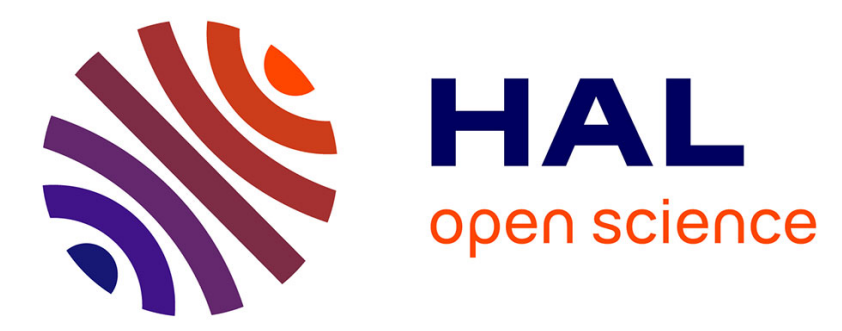

\title{
Single-Shot Femtosecond Spectroscopy of Reactive Organic Molecular Crystals
}

W. Wang, D. N. Chung, J. Fourkas, L. Dhar, K. Nelson

\section{To cite this version:}

W. Wang, D. N. Chung, J. Fourkas, L. Dhar, K. Nelson. Single-Shot Femtosecond Spectroscopy of Reactive Organic Molecular Crystals. Journal de Physique IV Proceedings, 1995, 05 (C4), pp.C4-289C4-296. 10.1051/.jp4:1995423 . jpa-00253722

\section{HAL Id: jpa-00253722 https://hal.science/jpa-00253722}

Submitted on 1 Jan 1995

HAL is a multi-disciplinary open access archive for the deposit and dissemination of scientific research documents, whether they are published or not. The documents may come from teaching and research institutions in France or abroad, or from public or private research centers.
L'archive ouverte pluridisciplinaire HAL, est destinée au dépôt et à la diffusion de documents scientifiques de niveau recherche, publiés ou non, émanant des établissements d'enseignement et de recherche français ou étrangers, des laboratoires publics ou privés. 


\title{
Single-Shot Femtosecond Spectroscopy of Reactive Organic Molecular Crystals
}

\author{
W. Wang, D.D. Chung, J.T. Fourkas, L. Dhar and K.A. Nelson \\ Department of Chemistry, Massachusetts Institute of Technology, Cambridge, MA 02139, U.S.A.
}

\begin{abstract}
$\underline{\text { Abstract }}$
Recent progress in single-shot femtosecond spectroscopy is described. This methodology is necessary for ultrafast time-resolved observation of solid-state chemical reactions, since the buildup of reaction products which cannot be flowed away or otherwise conveniently removed causes spectroscopic signals to change (and samples to undergo deterioration) as a particular region of the sample is irradiated many times. The first single-shot femtosecond observations of molecular vibrations and of short-time response in a photoreactive crystal are discussed.
\end{abstract}

\section{INTRODUCTION}

The mechanisms of chemical reactions in energetic solids have come under intense study in recent years. Advances in theory and simulation have provided models for the dynamics of reaction initiation, propagation, and termination, with important insights into the disposition of energy among collective and molecular vibrations and electronic degrees of freedom. Unfortunately, there is scant direct experimental guidance for and testing of the models. Most spectroscopy of real energetic materials and their chemical intermediates is done at very low temperatures [1], where the lifetimes of metastable species may become long, on nanosecond or slower time scales [2]. However, under ordinary conditions, many of the initial energy transfer and chemical events which may lead to detonation take place on femtosecond and picosecond time scales. According to differing models [3], these fast events may include transfer ("uppumping") of lattice vibrational energy to molecular vibrational or electronic degrees of freedom and early chemical events such as breaking (and in some cases forming) of covalent molecular bonds.

The experimental capabilities for femtosecond time-resolved spectroscopy of chemical reactions in gas and liquid phases are now well developed. Several femtosecond time-resolved measurements of reversible solid-state reactions, such as excimer formation in organic molecular crystals, have been reported. However, irreversible solid-state chemistry of the type occurring in energetic materials and many other reactive solids has never before been examined directly in the time domain. This has led to a dearth of experimental information about solid-state chemical reaction dynamics.

The experimental difficulty presented by solid-state photochemistry is the buildup of reaction products which cannot be flowed or otherwise conveniently removed from the region of optical observation. In contrast, gases and liquids can be easily flowed so that there is a continual replenishing of the irradiated volume with fresh sample material. As a result, the reaction products produced by the excitation pulse do not remain present to contribute to signal after successive excitations. In photoreactive crystals, the 
accumulation of reaction products can significantly influence measurements even after only a few excitations (sometimes just one excitation shot), and in many cases the irradiated region of the crystal cracks and even decomposes after a modest number of shots.

To circumvent these problems, we have developed an experimental methodology which permits femtosecond time-resolved measurements to be completed in a single laser shot. This capability is acquired easily on slower time scales, where an excitation pulse can be followed by a cw or quasi-cw probe beam whose time-dependent transmission through the sample may be resolved by a streak camera (picosecond time scales) or a wide-bandwidth oscilloscope (nanosecond time scales). There are no electronic components or data acquisition devices which can operate on femtosecond time scales, and so the method we have developed is all optical in nature. Here we present a description of the method and preliminary data from a molecular liquid and a photoreactive organic molecular crystal.

\section{SINGLE-SHOT FEMTOSECOND PUMP-PROBE SPECTROSCOPY}

In conventional pump-probe spectroscopy, two focussed laser beams are crossed in a sample; one beam acts as the excitation ("pump") pulse and the other as the probe pulse. In order to scan the temporal response of the sample, the time at which the probe pulse arrives at the sample can be varied with respect to the pump pulse by varying the distance the probe pulse must travel before arriving at the sample. This method, though, requires multiple pump-probe cycles in order to acquire a complete set of data points covering the desired temporal range. Each pump-probe cycle provides only one point on the time axis. This is clearly unacceptable when examining an irreversibly photoreactive solid.

Our single-shot technique, in contrast to the conventional pump-probe experiment which retrieves the array of temporal data points with an array of variably delayed probe pulses, each of which follows a different excitation pulse, retrieves the temporal information with a single cylindrically focussed probe pulse. The approach [4] is illustrated in Fig. 1. In this method, both the pump and probe beams are cylindrically focussed such that they overlap with each other along a line at the sample. In addition, the beams are arranged such that they overlap at a substantial angle (typically about $25^{\circ}$ ) relative to each other. As illustrated in the Fig. 1, the pump pulse wavefront may arrive normal to the sample so that the entire irradiated region is excited simultaneously. Since the probe pulse is incident on the sample at a substantial angle away from the normal, different regions of the probe pulse arrive at the sample at different delay times relative to the excitation pulse. Consequently, the different parts of the probe beam are able to retrieve information about the sample's temporal response at different times. A CCD array detector is then used to measure the spatially encoded temporal response by measuring the intensity of the transmitted probe pulse along the cylindrically focussed line. Figuratively, the CCD array spatially resolves the single beam into an apparent array of probe pulses. Each element in the $\mathrm{CCD}$ array conveys a data point at a distinct time delay relative to the excitation pulse, and all the elements in the array together describe the response of the sample over an extended temporal range. 

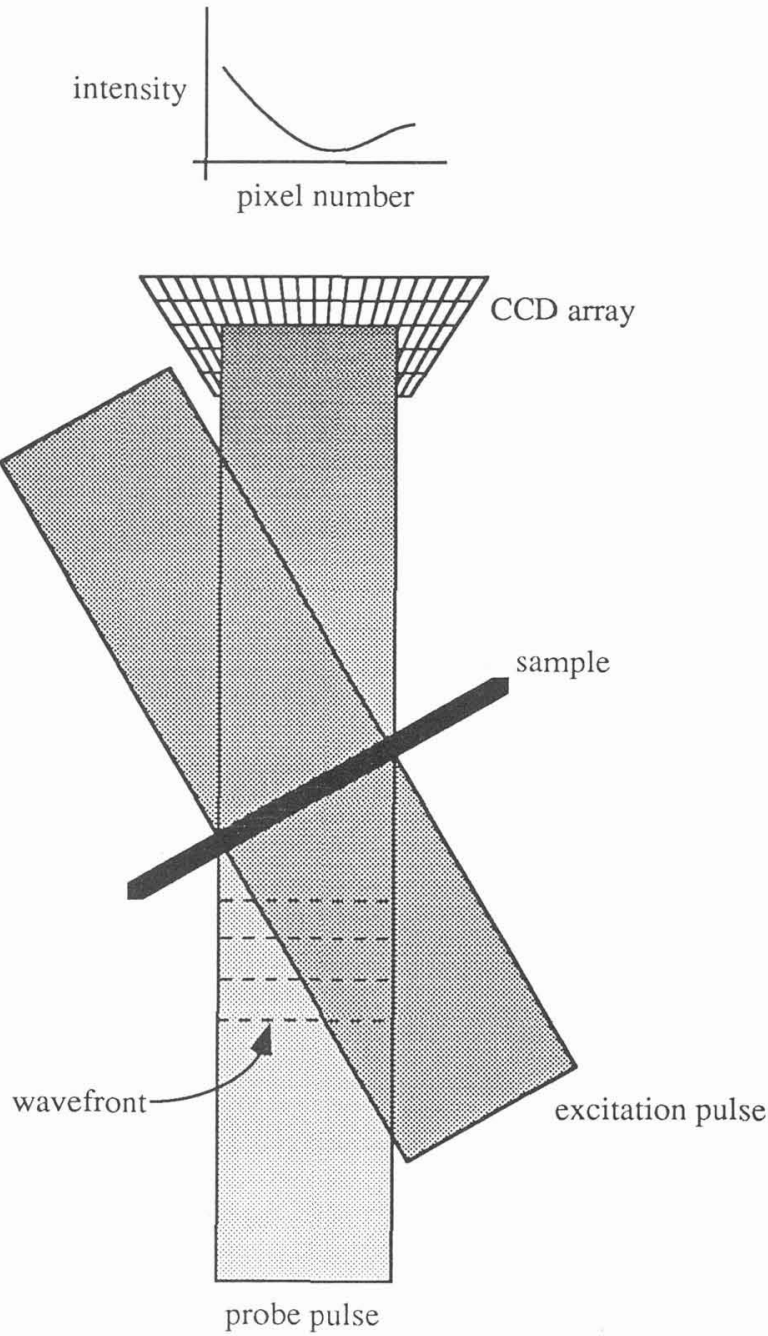

Figure 1: Schematic illustration of the single-shot technique 


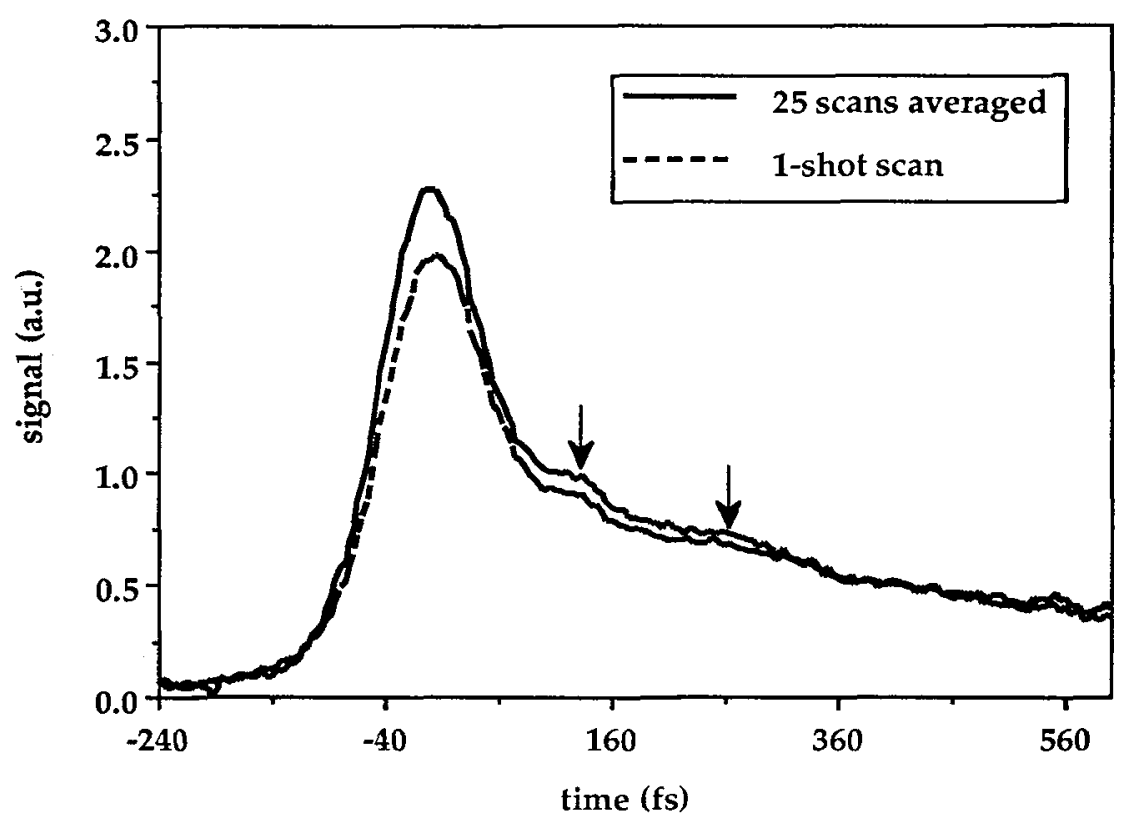

Figure 2: A transient absorption single-shot scan of ethyl violet in methanol compared to a 25 -shot scan. The arrows indicate where the oscillations can be seen.

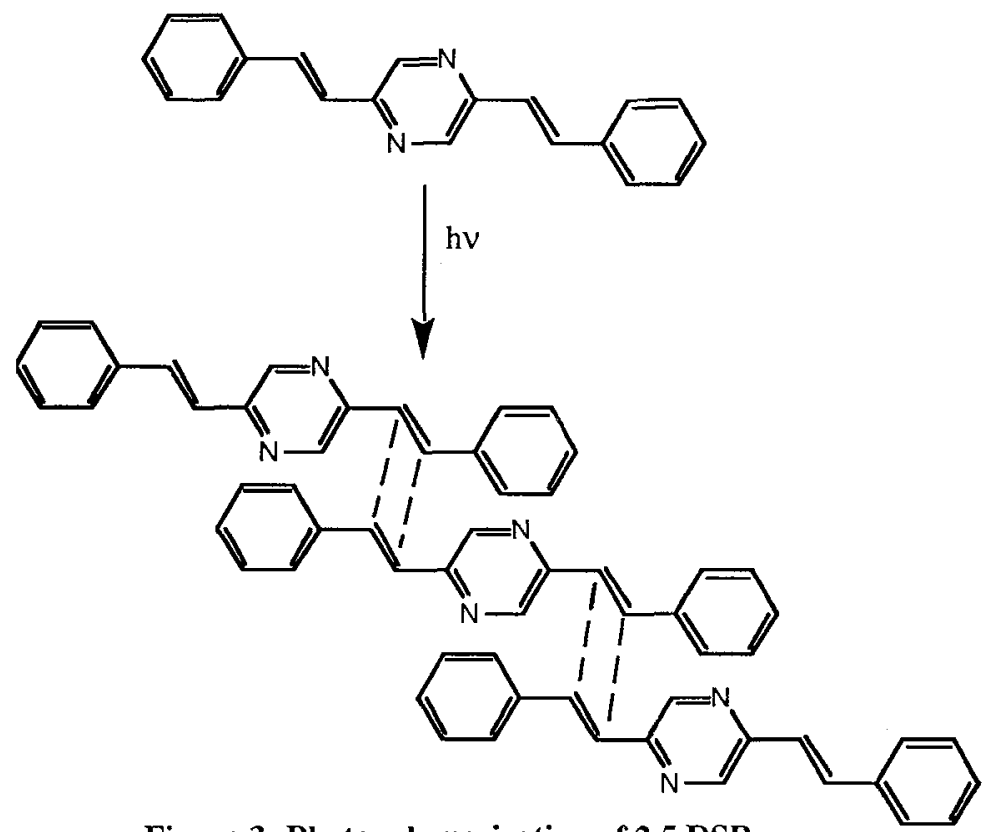

Figure 3: Photopolymerization of 2,5 DSP 


\section{REAL-TIME OBSERVATIONS OF MOLECULAR VIBRATIONS IN LIQUID SOLUTIONS}

To demonstrate the single-shot technique, we first examined the relaxation dynamics of ethyl violet in methanol which has been already studied by conventional pump-probe spectroscopy [5]. The laser system used to conduct this experiment has been described in detail in an earlier publication [6]. Briefly, the femtosecond excitation and probe pulses at $620 \mathrm{~nm}$ were generated by a sync pumped antiresonant ring dye laser which was pumped by the frequency-doubled output of a mode-locked Nd:YAG laser. The dye laser output was amplified in a three-stage amplifier chain which was pumped by a $\mathrm{Nd:YAG} \mathrm{regenerative}$ amplifier. A mechanical chopper was used to reduce the pulse repetition such that the shutter on the CCD was able to sample only one pulse. The pulse width of the pump and probe was measured to be $72 \mathrm{fs}$.

From the earlier study done by conventional pump-probe spectroscopy, it was found that with a sufficiently short pulse, ethyl violet dye exhibits a 155 -fs oscillation. When the dye molecule is excited, the phenyl groups in the dye molecule rotate to a new conformation in the excited state. It is believed that the observed oscillations reflect coherent vibrational motion of the dye molecules which occurs in the electronic excited state reached through optical absorption. Encouragingly, the oscillations in Fig. 2 were found to match the vibrational period measured by conventional femtosecond pump-probe spectroscopy. Furthermore, the signal-to-noise ratio between scans from a single-shot data set and from a 25 -shot data set were not significantly different.

\section{PRELIMINARY RESULTS FROM A PHOTOREACTIVE CRYSTALLINE SOLID}

Encouraged by the liquid-state results, we began a study of the photoreactivity of a molecular crystal, 2,5-distyrylpyrazine (DSP). DSP is a diolefin which undergoes $[2+2]$ photoaddition at both olefinic positions when irradiated with UV light (Fig. 3) [7,8]. DSP crystal structure plays an important role in dictating its reactivity. It is known that DSP crystallizes in two crystallographic forms, one is photoreactive while the other is photostable. The reactivity difference was attributed to the different molecular packing in the two polymorphs. When the photoreactive DSP crystal is irradiated with light of wavelength longer than about $400 \mathrm{~nm}$, it forms oligomers with an average length of three monomer units. When it is further irradiated with light below $400 \mathrm{~nm}$, the oligomers will polymerize.

While extensive studies have been done on DSP in the crystalline state [8], no ultrafast time-resolved study has been performed. In our experiment, we used a mode-locked titanium:sapphire laser (Coherent) pumped by an argon-ion laser (Coherent). The pulses from that laser were amplified through chirped-pulse amplification by a home-built regenerative amplifier [9] pumped by a home-built Q-switched intracavityfrequency-doubled Nd:YAG laser. The amplified output at $810 \mathrm{~nm}$ was used as the probe pulse, and the frequency-doubled light at $405 \mathrm{~nm}$ was used as the excitation pulse. The pulsewidth was typically $150 \mathrm{fs}$.

The data collected from DSP in solution (chloroform) using the single-shot technique were found to be congruent to the data collected using the conventional pump-probe technique (Fig. 4). In the crystal, the almost parallel plane-to-plane stacking of the molecules should predispose it toward faster reaction than in solution. Surprisingly, it was found (Fig. 5) that the temporal response of DSP in crystalline form was identical to the temporal response of the molecule in solution in the 2-ps temporal range examined. In view of the high photochemical quantum yield of DSP [10], the primary pathway for population decay from the excited state should come from photoaddition and not from radiative or non-radiative energy transfer. Assuming the signal decay is proportional to the decay of the excited-state population from reaction, it appears, from the absence of any signal decay, that DSP does not react within the first 2 ps of irradiation. 


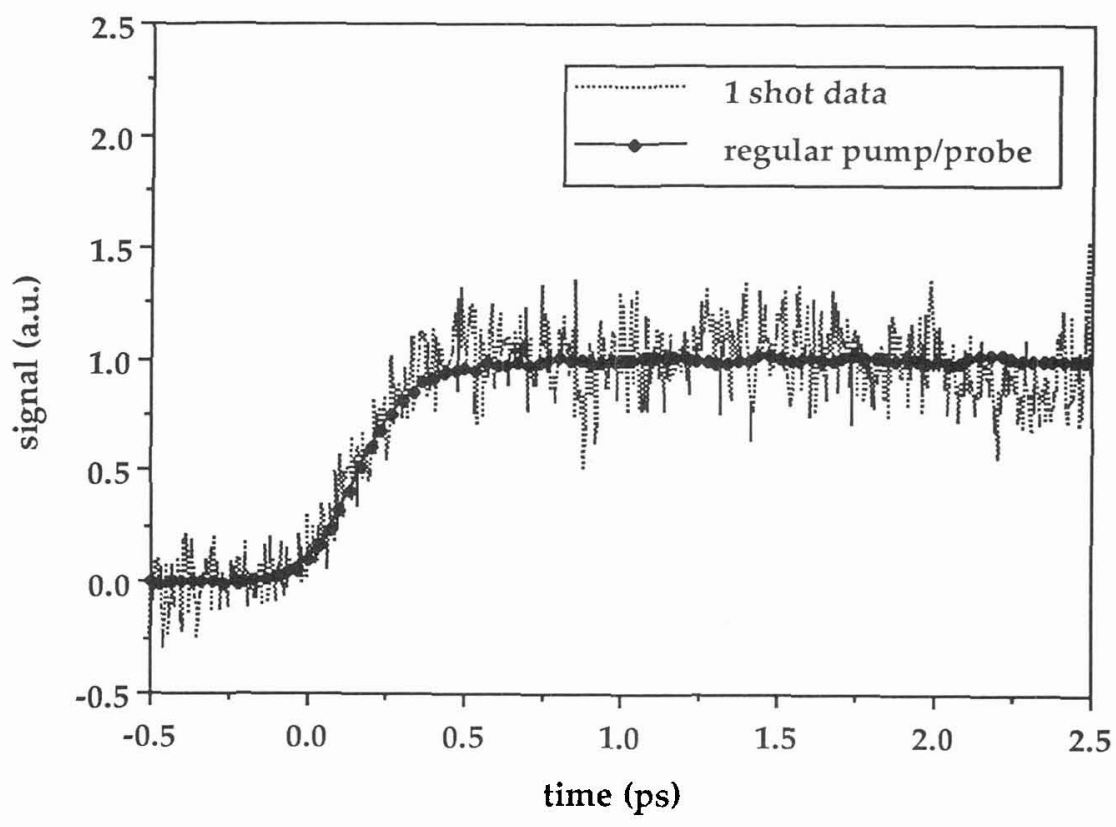

Figure 4: Single shot scan compared to a conventional pump-probe scan of 2,5 DSP in solution

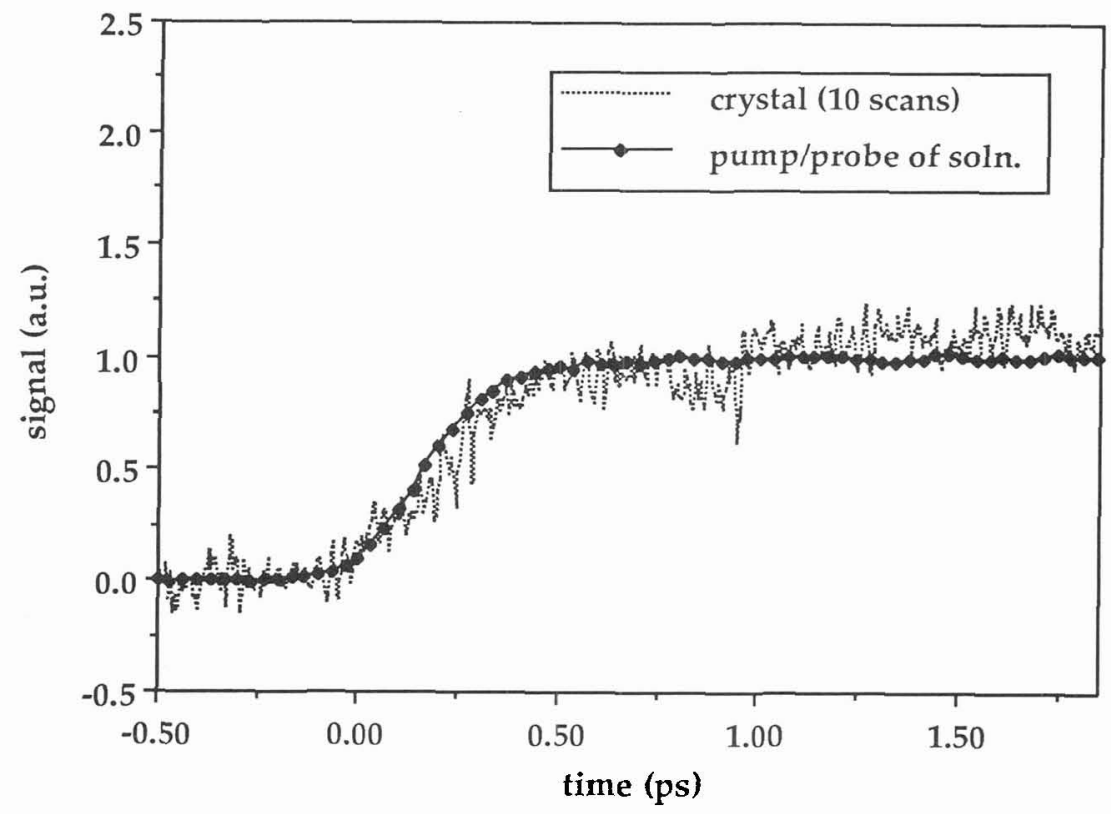

Figure 5: 10 averaged single shot scans of a 2,5 DSP crystal compared to conventional pump-probe data of 2,5 DSP in chloroform 
When crystalline DSP is excited by light of wavelengths longer than $400 \mathrm{~nm}$, two transitions, $\pi * \leftarrow \pi$ and $\pi * \leftarrow \mathrm{n}$, are both excited. The non-bonding electron in the nitrogen from the pyrazine ring is excited in the $\pi * \leftarrow \mathrm{n}$ transition while the electron in the highest occupied $\pi$ molecular obital is excited by the $\pi * \leftarrow$ $\pi$ transition. It is believed that the exciton formed from the $\pi * \leftarrow n$ transition, which is localized and shows strong exciton-phonon coupling, helps to trap the delocalized exciton from the $\pi * \leftarrow \pi$ transition and thereby facilitate the photoaddition at the double bonds on the styryl moiety [11]. In solution, photodimerization is expected to be slow since monomers must encounter each other to react. In the crystal, reaction might have been expected to occur on a faster time scale. There are at least two possible reasons why this reaction doesn't happen on a femtosecond time scale. First, localization of the initially excited exciton state may take longer than 2 ps. Second, even if the trapping process does occur quickly, it is possible that there is a potential energy barrier to molecular motion along the reaction pathway from the initial configuration to a higher-energy transition state and finally to the product.

Further experiments are under way to gain a deeper understanding of the DSP system. It is noteworthy as a demonstration of experimental capabilities that the irradiated regions of this material not only have a buildup of reaction product but undergo mechanical shattering and decomposition after just a few seconds of irradition at a $1-\mathrm{KHz}$ repetition rate. This illustrates graphically the need for a single-shot experimental approach.

\section{OUTLOOK}

The study of ultrafast reaction dynamics in energetic solids requires single-shot experimental methods. We have demonstrated a suitable approach through measurements of liquid-state molecular vibrations and preliminary measurements of the short-time behavior of a photoreactive solid. Experiments on energetic materials are now in preparation. Measurements of solid-state chemical reaction dynamics promise to offer fundamental insight into the effects of the nonreactive surroundings in any phase. In energetic materials, the crucial effects of the surroundings in mediating energy exchange among reactive and nonreactive channels will be opened up for study through direct time-resolved observation of reaction dynamics and energy relaxation processes.

This work was supported in part by ONR Grant No. N00014-90-J-4070. DDC thanks the National Science Foundation for a predoctoral fellowship in chemistry. We thank N.M. Peachey (Los Alamos National Laboratory) and C.J. Eckhardt (University of Nebraska-Lincoln) for providing us the DSP crystals used in this study. We thank Yongqin Chen (University of California-Berkeley) for providing us a design of the Qswitched intracavity doubled Nd:YAG laser and Jeff Squier (University of Michigan) for help on the Ti:Sapphire amplifier.

\section{REFERENCES}

1. J.M. McBride and J.P. Toscano. ONR/LANL Workshop Fund. Phys. and Chem. of Combustion, Initation, and Detonation of Energetic Materials. (CPIA Publ. 589, March 1992), 71.

2. Y.M. Gupta. In Shock Compression in Condensed Matter 1991. S.C. Schmidt, Ed. North-Holland: Amsterdam, Netherlands, (1991), 15. 
3. S.Chen, W.A. Tolbert, and D.D. Dlott. J. Phys. Chem. 98 (1994) 7759; L.E. Fried and A.J. Ruggiero. J. Phys. Chem. 98 (1994) 9786.; A. Tokmakoff, M.D. Fayer, and D.D. Dlott. J. Phys. Chem. 97 (1993) 1901; G. Gao, R. Pandey, and A.B. Kunz. In Structures and Properties of Energetic Materials. D.H. Liebenberg, R.W. Armstrong, and J.J. Gilman, Eds. Materal Research Society: Pennsylvania, (1993), 149. J.J. Gilman. Philos. Mag. B. 67 (1993) 207. T. Luty and R. Fouret. J. Chem. Phys. 90 (1989) 5697. F.J. Zerilli and E.T. Toton. Phys. Rev. B. 29 (1984) 5891.

4. L. Dhar, J.T. Fourkas, and K.A. Nelson. Optics Letters. 19 (1994) 1.

5. F.W. Wise, M.J. Rosker, and C.L. Tang. J. Chem. Phys. 86 (1987) 2827.

6. S. Ruhman, A.G. Joly, B. Kohler, L.R. Williams, and K.A. Nelson. Revu de Phys. Appl. 22 (1987) 1717.

7. M. Haswegawa, in Advances in Polymer Science, Volume 42, Springer-Verlag Berlin, Heidelberg, 1982. p.1.

8. H. Nakanishi, Y. Suzuki, F. Suzuki, and M. Hasegawa. J. Polym. Sci. A-1, 7 (1969) 753; J. Swiatkiewicz, G. Eisenhardt, P.N. Prasad, J.M. Thomas, W. Jones, and C.R. Theocaris. J. Phys. Chem. 86 (1982) 1764; E.M. Ebeid, and S.E. Morsi. J. Chem. Soc. Faraday Trans. I. 79 (1983) 1183.; E.M. Ebeid and N.J. Bridge. J. Chem. Soc. Faraday Trans. I. 80 (1984) 1113; J. Swiatkiewicz and P.N. Prasad. J. Polym. Sci: Polym. Phys. Ed. 22 (1984) 1417; N.M. Peachey and C.J. Eckhardt. Chem. Phys. Lett. 188 (1992) 462; N.M. Peachey and C.J. Eckhardt. J. Phys. Chem. 98 (1994) 7106.

9. F. Salin, J. Squier, G. Mourou, and G. Vaillancourt, Opt. Lett. 16, 1964(1993).

10. E.M. Ebeid, M.H. Abdel-Kader, and S.E. Morsi. J. Chem. Soc. Faraday Trans. I. 78 (1982) 3213.

11. N.M. Peachey and C.J. Eckhardt. J. Am. Chem. Soc., 115 (1993) 3519. 
Questions - Answers, Comments

\section{Dlott - Califano :}

Q : How can your formalism be extended to consider localized phenomena such as the passage of a sharp shock front through a crystal?

$\mathbf{A}$ : The scattering process is localized at every site where are impurities. What is delocalized is the concept of phonons. This concept of phonons is non localized because in the theory one develops any local transition in terms of phonons of the original crystal, as a basis functions. But the process is localized.

\section{Rullière - Nelson :}

Q : Gaussian beam shape leads to an inhomogeneous repartition of the excited state population. In single shot technics, this inhomogeneous repartition can lead to under/overestimation of the measured rates. How do you manage this problem?

A : We examine the probe beam spatial profile very carefully on a single-shot basis, using a "reference" beam which reaches our CCD detector just like the transmitted "probe" beam. Separately, we examine the excitation pulse spatial profile - usually averaged over several shots. In practize we can now work with precision of about $0.1 \%$. Ultimately our ability to measure small changes in the sample's absorption (i.e. small changes in probe transmission) is limited by how precisely we can characterize the beam profiles.

$\mathbf{Q}$ : With large spectral range probe beams it is very difficult to avoid the "chirp" problem. It means that along the beam diameter of the excitation beam, you will not have, for a given position, the same time reference for all wavelengths. How do you take into account this problem?

A : In proposed experiments we will resolve both time and frequency, yielding a broadband absorption spectrum as a function of time in a simple shot. In this case "chirp", in which for example the redder part of the pulse arrives at the sample before the bluer part, presents a problem. Of course, as much as possible we'll eliminate chirp so that each part of the pulse has the same spectrum. But in any event, we must still use a "reference" pulse which, like the transmitted probe pulses is displayed in two dimensions (one for time and one for wavelength) on the CCD. In this manner we can correct for any chirp. As in the current 1-dimensional case (a "line" is displayed on the $\mathrm{CCD}$, different points corresponding to different times), our precision will depend on how well we can characterize the reference beam and normalize the signal beam.

\section{Melius - Nelson :}

$\mathbf{Q}$ : Is it possible to use your multi-pulse Raman pumping to do state-selected intramolecular vibrational excitation (e.g. a C-H stretch or an $\mathrm{O}-\mathrm{H}$ stretch)?

A : Using ISRS as the excitation mechanism, no - because the frequency is too high. The pulse duration has to be short compared to one vibrational cycles and that would mean just a few 
femtosecond in this case. But it is possible to convert visible fs pulses to IR fs pulses. These can drive polar modes directly, an IR pulse sequences can be made. In this way it may be possible to drive large $\mathrm{OH}$ amplitudes, for example.

\section{Dufort - Nelson :}

$\mathbf{Q}$ :What are the devices used to achieve wavelength conversion in your IR experiments ?

A : A difference-frequency mixing crystal can be used to convert a visible femtosecond pulse to an IR femtosecond pulse. If the IR pulse duration is too long then another (sum) frequency mixing crystal can be used to go back to the visible. This mixer can be gated by an ultrashort visible pulse to preserve good time resolution.

$\mathbf{Q}$ : What are the methods today - on a theoretical point of view - to investigate what will be the shifts of the vibration modes of the molecules under extreme conditions of pressure or (and) temperature of the detonation processes?

A : At static pressure, not in real shock wave, it requires expertise but it is doable. To do it under shock loading requires extraordinary expertise and experimental apparatus that Dr Gupta would be able to do.

$\mathbf{Q}$ : In the reactive zone to analyse process of decomposition, among different patterns knowledge of the evolution of vibrational molecules is needed. How to do?

A : Real spectroscopy in the reaction zone, we have not already a good solution.

Bratos : Comment In extreme conditions of high temperature and pression, the vibrational normal mode is no more a useful concept. Moreover, the distribution of vibrational modes is quasi continuous. In these conditions, the analytical treatments are particularly difficult, and numerical methods seem appropriate.

Dremin : We study shock energy transfer to molecules. Shock excitation is not the same process than laser excitation.

Under compression, even through a weak shock, EMs behaves like a liquid, and from an energetic point of view like a chaos. Is it possible to use the phonon concept for this state of the material ? About chemical reactions, we are interested with the very very beginning of chemical change, shock mechanical energy transfer to EM. How does it proceed?

\section{Kondrikov - Nelson :}

Q : Using one shot excitation you obtain a vibrational mode of a bond in the molecule, and the amplitude of the vibration is about 1-100 fm, essentially harmonic approximation. When you use your mechanism of multisteps excitation, the amplitude enhences in $10^{3}-10^{4}$ times, and consequently you can include into the move all the neighboring molecules, so you transfer another vibrational, and breaking process. Am I right?

A : First a clarification. Our "single-shot" method is for detection, not excitation. It allows us to do 
experiments on irreversible events in crystals without warrying about the effects of permanent change on the "next" laser shot, which never comes. It's separate from ISRS excitation of lattice phonons, using single or mutiple pulses.

Concerning ISRS excitation, if we can reach large amplitudes we will certainly reach the anharmonic region with increased mode-mode coupling. The issue isn't coupling between one molecule and the others, since we are mainly concerned with collective (optic phonon) modes anyway, but coupling among different optic phonon and also molecular (vibron) modes. There are important non linear lattice dynamics effects that need to be considered, so that our optimum pulse sequence probably will not consist simply of evenly spaced pulses as in the harmonic limit. I think that pulse sequences will provide a factor of more than 10 on vibrational amplitude in many cases (resonance enhancement and higher pulse intensities that are possible on a single-shot basis will also help). With all this we should reach the 0.1-0.5 Angstrom vibrational amplitude regime in some cases.

\section{When a rest is needed}

\section{Dufort - Nelson}

Q : Pr Nelson, what is your opinion of the french school of Saussure, Foucault, Lacan, Derrida et al?

Going back farther, could you comment on Rousseau versus Sade ?

A : Thank you for this opportunity. Rousseau was the father of modern Romantism, what essentially defines modern-day personality in the west. Unfortunately, he is also remembered for his proposal that man is inherently good and evil acts are due only to corrupting influences of society. This neglects deep-rooted anxiety and turbulence inherent in human character, in my view. The Marquis de Sade opposed this view by celebrating man's darker nature - the inevitable progression from Romantism to Decadence.

Foucault, Lacan et al. built on Rousseau's weak premises adding the central idea that all experience is constituted by language. This further disregards the complexity, turbulence and possibility for heroic struggle against the self that pervades human subconscious and conscious in my view. 


\section{MOLECULAR MECHANISMS OF ENERGY TRANSFER IN EMS}

Chairman : Jacques Boileau, Expert DRET

I am in charge to introduce the lectures about mechanisms of energy transfers and molecular responses in energetic materials.

Generally speaking, the goal is to find a high explosive with high performances, associated with a high degree of safety and a reasonable cost. It must detonate under a programmated and strong enough initiation, but also remain quiet and without severe reactions under untimely stimuli. A way is to understand the SDT mechanisms of energetic materials.

\section{General remarks}

11. In the mechanisms, it is necessary to distinguish initiation and propagation of detonation $:$ it is not always clear in the theories.

The initiation may occur by shock, and also by laser.

- It is necessary to define what is a shock : pressure, duration, eventually the delay to obtain the pressure. Then, what is the signification of a shock at a molecular size? What is a shock on a crystal or on a liquid?

- The effect of a laser pulse to initiate an energetic material can be a direct interaction with the molecules, or an intermediate initiation of a slapper. The mechanisms may perhaps be different, but it could exist a synchronization problem for the observation.

12. The sequence of successive events delays is important : subpico, pico, nano, microseconds. Do not forget these time scales in a range of 10 power 6 or 7 (the same ratio than between 1 second and 3 months). In this frame, some observations may be optical (laser) and/or electrical ones, e.g. emission or absorption of light...

Theories must be compatible or coherent with the observations and the experiments results. It is absolutely necessary, when a theory is presented, to imagine the simple, and, if possible, cheap and easy to build, experiments that may ascertain or invalidate the theory. The theory has to be able to explain the observations. Don't forget that theories may be correct in some domains and not in others. Sometimes, or often, some theories are simultaneously valuable.

\section{Theories on detonation for macroscopically homogeneous explosives}

They may be divided into two groups of mechanisms.

- Heterogeneous mechanisms : hot spots generated by voids and cavities, by shearing, by crystal defects, by steric hindrance effects (Armstrong, Coffey, Dick ..).

- Homogeneous mechanisms. 
The discussion is : how the shock energy goes into the molecules and the crystalline lattice?

The beginning mechanism is totally out of equilibrium. Molecules and atoms motions are quantified. Some theories (Fayer, Dlott, Tarver...) suppose a transfer to vibration modes without push of the molecules into excited electronic states.

Some other mechanisms suppose transitions to electronic states (e.g. predissociative) (Delpuech, Odiot, Sharma...)

The role of free radicals in the shock front has been emphasized by $F$.Walker.

An effect of bond breaking by shock has been proposed by Gilman; he supposes also that the electrons ejected by this break form a plasma that plays a role.

Tang proposes a mechanism where the shock creates inhomogeneities similar to hot spots.

Dremin distinguishes domains for both heterogeneous and homogeneous mechanisms, giving an explanation about the energy transfer phases.

You will find successively the explanations given by S.Dufort, F.Walker, D.Dlott and A.Dremin.

I hope that the discussion allows to explain some observations : For instance :

- The results described in the thesis of Spitzer on PETN monocrystals, for instance the light emission during the detonation process

- Why is it no evident correlation between sensitivity and critical diameter (e.g. HNS)?

- Why some high explosives are more sensitive in a liquid than in a solid state (e.g. hydrazine nitrate, perhaps TNT) and the reverse for others $\left(H N F_{2}\right.$, perhaps nitroglycerine)?

- Other questions : are the mechanisms different for the same product in solid and in liquid phase?

Don't forget also that sensitivity is a macroscopic observation which includes the measurement methods together with the characteristics of the crystals.

Les méthodes prédictives pour le développement de nouvelles molécules explosives ; S.Dufort

A new Kinetics and the Simplicity of Detonation ; F.E.Walker

Molecular Mechanical Energy Transfer behind the Shock Front - see 3-1 paper, Theoretical Model ; D.D.Dlott

EM Shock Wave Chemistry, see paper, Towards Detonation Theory, § 4 ; A.N.Dremin 